\title{
On Definable Scale Reduction by Simultaneous Observations ${ }^{1}$
}

\author{
J. J. Stephens \\ Dept. of Meleorology, Florida State University, Tallahassee \\ (Manuscript received 7 September 1970, in revised form 16 October 1970)
}

\begin{abstract}
A two-dimensional extension of the Sampling Theorem with the inclusion of derivative samples is presented. It is shown that simultaneous sampling of geopotential and horizontal wind components on a mesh with elements $\Delta x, \Delta y$ permits definition of waves of length $\Delta x$ and $\Delta y$ along mesh lines under the geostrophic assumption. Additional independent estimates of either of the horizontal shear elements, $\partial u / \partial x$ or $\partial v / \partial y$, permits the exact reconstruction of band-limited fields whose spectral decomposition includes waves of length $\Delta x$ and $\Delta y$, or longer.
\end{abstract}

\section{Introduction}

Definitive observational investigation of atmospheric structure includes sufficient samples to identify all significant scales, such as might be reflected in the variance spectrum. Otherwise, aliasing will obscure the true structure. Based on the analysis of band-limited functions, the minimum definable wavelength is twice the sampling interval. As shown by Linden and Abramson (1960) for functions of one independent variable, the addition of derivative information reduces the definable scale. The implication of simultaneously sampling several dependent fields linked by an equation of constraint is that a smaller scale can be defined. While this has been understood intuitively, no quantitative statement of the scale reduction has been given for various field combinations.

The economics of definable scale reduction for certain combinations of field observations is important. For instance, a reduction of one-half in the definable scales in both coordinates in the plane by virtue of the appropriate combination of field observations leads to a fourfold decrease in the number of observing sites. From another viewpoint, for given observational arrays linked by equations of constraint, it will be possible in some instances to redefine them on a finer mesh. This could be accomplished with Sasaki's (1958) method.

A two-dimensional analogue of the generalized Sampling Theorem (Linden and Abramson, 1960) is proven here to demonstrate that simultaneous sampling of geopotential $\Phi$ and horizontal wind components $u$ and $v$ on a mesh with intervals $\Delta x$ and $\Delta y$ defines waves of length $\Delta x$ and $\Delta y$ along mesh lines under the geostrophic assumption. If independent estimates of either of the horizontal shear elements, $\partial u / \partial x$ or $\partial v / \partial y$, are available, then the fields can be reconstructed exactly if their

${ }^{1}$ Research supported by the U. S. Army Electronics Command, Contract No. DAAB07-69-C-0062, under Project THEMIS. spectral decomposition consists of waves no shorter than $\Delta x$ and $\Delta y$ in the $x$ and $y$ directions, respectively.

The theorem is also applicable to temperature observations in the plane, with horizontal gradient estimates supplied by wind shear observations through the thermal wind equation.

\section{A two-dimensional sampling theorem}

Let $\Phi(x, y)$ be an absolutely integrable band-limited function with continuous second derivatives whose Fourier transform $F(m, n)$ vanishes for $|m|>M=2 \pi / \Delta x$ and $|n|>N=2 \pi / \Delta y$, where $M$ and $N$ are positive radian wavenumbers. Then

$$
\begin{array}{r}
\Phi(x, y)=\sum_{k=-\infty}^{\infty} \sum_{l=-\infty}^{\infty}\{\Phi(k \Delta x, l \Delta y) \\
+(x-k \Delta x) \Phi_{x}(k \Delta x, l \Delta y)+(y-l \Delta y) \Phi_{y}(k \Delta x, l \Delta y) \\
\left.+(x-k \Delta x)(y-l \Delta y) \Phi_{x y}(k \Delta x, l \Delta y)\right\} \\
\quad \times\left\{\operatorname{dif}\left(\frac{M}{2} x-k \pi\right) \operatorname{dif}\left(\frac{N}{2} y-l \pi\right)\right\}^{2},
\end{array}
$$

where

$$
\operatorname{dif}(x)=\frac{\sin x}{x}
$$

is the diffraction (cardinal, composing) function. Partial derivatives are indicated by subscripts. Thus, $\Phi(x, y)$ is exactly reconstituted, including contributions from waves of length $\Delta x$ or $\Delta y$, by observations of $\Phi, \Phi_{x}, \Phi_{y}$ and $\Phi_{x y}$ on the mesh points $(k \Delta x, l \Delta y)$. As shown by Stephens (1966), a similar theorem for samples of $\Phi(k \Delta x, l \Delta y)$ alone allows identification of waves $2 \Delta x$ or $2 \Delta y$, or longer. 


\section{Theorem proof}

A direct proof of (1) is given here. It will be shown that the Fourier transform, say $\hat{F}(m, n)$, of (1) is equal to $F(m, n)$. The two-dimensional Fourier transform pair used here is

$$
\begin{aligned}
& F(m, n)=\text { F.T. }[\Phi(x, y)] \\
& =\frac{1}{2 \pi} \int_{-\infty}^{\infty} \int_{-\infty}^{\infty} \Phi(x, y) e^{-i(m x+n y)} d x d y, \\
& \Phi(x, y)=\mathrm{F} . \mathrm{T} \cdot{ }^{-1}\{F(m, n)\} \\
& =\frac{1}{2 \pi} \int_{-\infty}^{\infty} \int_{-\infty}^{\infty} F(m, n) e^{i(m x+n y)} d m d n .
\end{aligned}
$$

Since

$$
\begin{aligned}
\text { F.T. }\left\{\left[\operatorname{dif}\left(\frac{M}{2} x-k \pi\right) \operatorname{dif}\left(\frac{N}{2} y-l \pi\right)\right]^{2}\right\} \\
=S(m, n) e^{-i(m k \Delta x+n l \Delta y),}
\end{aligned}
$$

$$
\begin{array}{r}
\text { F.T. }\left\{(x-k \Delta x)\left[\operatorname{dif}\left(\frac{M}{2} x-k \pi\right) \operatorname{dif}\left(\frac{N}{2} y-l \pi\right)\right]^{2}\right\} \\
=i S_{m}(m, n) e^{-i(m k \Delta x+n l \Delta y)} ;
\end{array}
$$

F.T. $\left\{(y-l \Delta y)\left[\operatorname{dif}\left(\frac{M}{2} x-k \pi\right) \operatorname{dif}\left(\frac{N}{2} y-l \pi\right)\right]^{2}\right\}$

$$
=i S_{n}(m, n) e^{-i(m k \Delta x+n l \Delta y)} \text {, }
$$

F.T. $\{(x-k \Delta x)(y-l \Delta y)$

$$
\begin{aligned}
\left.\times\left[\operatorname{dif}\left(\frac{M}{2} x-k \pi\right) \operatorname{dif}\left(\frac{N}{2} y-l \pi\right)\right]^{2}\right\} \\
=-S_{m n}(m, n) e^{-i(m k \Delta x+n l \Delta y)},
\end{aligned}
$$

where the subscripts again indicate partial derivatives,

$S(m, n)= \begin{cases}\frac{2 \pi}{M^{2} N^{2}}[\operatorname{sgn}(m) m-M][\operatorname{sgn}(n) n-N] ; \\ 0 ; & |m|<M,|n|<N \\ & |m|>M,|n|>N\end{cases}$

and

$$
\operatorname{sgn}(m)=\left\{\begin{array}{rr}
1 ; & m>0 \\
-1 ; & m<0
\end{array}\right.
$$

the transform of (1) can be written as

$$
\begin{array}{r}
\hat{F}(m, n)=\sum_{k=-\infty}^{\infty} \sum_{l=-\infty}^{\infty} e^{-i(m k \Delta x+n l \Delta y)}\{S(m, n) \Phi(k \Delta x, l \Delta y) \\
+i S_{m}(m, n) \Phi_{x}(k \Delta x, l \Delta y)+i S_{n}(m, n) \Phi_{y}(k \Delta x, l \Delta y) \\
\left.-S_{m n}(m, n) \Phi_{x y}(k \Delta x, l \Delta y)\right\} .
\end{array}
$$

The infinite Dirac comb

$$
\nabla(x, \Delta x)=\Delta x \sum_{k=-\infty}^{\infty} \delta(x-k \Delta x)
$$

can be introduced to write (11) as

$$
\begin{aligned}
\frac{\Delta x \Delta y}{2 \pi} \hat{F}(m, n)=S(m, n) \mathrm{F} . \mathrm{T} .\{\Phi(x, y) \nabla(x, \Delta x) \nabla(y, \Delta y)\} \\
\quad+i S_{m}(m, n) \text { F.T. }\left\{\Phi_{x}(x, y) \nabla(x, \Delta x) \nabla(y, \Delta y)\right\} \\
\quad+i S_{n}(m, n) \text { F.T. }\left\{\Phi_{y}(x, y) \nabla(x, \Delta x) \nabla(y, \Delta y)\right\} \\
\quad-S_{m n}(m, n) \mathrm{F} . \mathrm{T} .\left\{\Phi_{x y}(x, y) \nabla(x, \Delta x) \nabla(y, \Delta y)\right\} .
\end{aligned}
$$

The two-dimensional convolution property for the transform pair used here is

F.T. $\{g(x, y) h(x, y)\}$

$$
=\frac{1}{2 \pi} \int_{-\infty}^{\infty} \int_{-\infty}^{\infty} H(k, l) G(m-k, n-l) d k d l,
$$

where $H(m, n)$ and $G(m, n)$ are the transforms of $h(x, y)$ and $g(x, y)$, respectively. Also,

$$
\begin{aligned}
& \text { F.T. }\{\nabla(x, \Delta x) \nabla(y, \Delta y)\} \\
& =2 \pi \sum_{k=-\infty}^{\infty} \sum_{l=-\infty}^{\infty} \delta(m-k M) \delta(n-l N), \\
& \text { F.T. }\left\{\Phi_{x}(x, y)\right\}=i m F(m, n), \\
& \text { F.T. }\left\{\Phi_{y}(x, y)\right\}=i n F(m, n), \\
& \text { F.T. }\left\{\Phi_{x y}(x, y)\right\}=-m n F(m, n) .
\end{aligned}
$$

Thus, (13) can be written as

$$
\begin{aligned}
& \frac{\Delta x \Delta y}{2 \pi} \hat{F}(m, n)=\sum_{k=-\infty}^{\infty} \sum_{l=-\infty}^{\infty} F(m-k M, n-l N) \\
& \quad \times\left\{S(m, n)-S_{m}(m, n)(m-k M)-S_{n}(m, n)(n-l N)\right. \\
& \left.\quad+S_{m n}(m, n)(m-k M)(n-l N)\right\} .
\end{aligned}
$$

Since $F(m, n)$ vanishes for $|m|>M$ or $|n|>N$, it follows that the only potential non-zero contributions to (19) are for $|k| \leqslant 1$ and $|l| \leqslant 1$. In particular, if $m<0$, only $k \leqslant 0$ contributes. If $m>0$, only terms with $k \geqslant 0$ enter. Similar considerations apply for $n$. The lengthy expansion and collapse of (19) will be demonstrated with the case $m>0$ and $n<0$. The expansion becomes

$$
\begin{aligned}
M N \hat{F}(m, n) & =F(m, n+N)\{-(m-M)(n+N) \\
& +m(n+N)+(m-M)(n+N)-m(m+N)\} \\
& +F(m, n)\{-(m-M)(n+N)+m(n+N) \\
& +(m-M) n-m n\}+F(m-M, n+N) \\
& \{-(m-M)(n+N)+(m-M)(n+N) \\
& +(m-M)(n+N)-(m-M)(n+N)\} \\
& +F(m-M, n)\{-(m-M)(n+N) \\
& +(m-M)(n+N)+(m-M) n-(m-M) n\} \\
& =M N F(m, n) .
\end{aligned}
$$


Similar expansions apply in the remaining wavenumber quadrants so that $\hat{F}(m, n)=F(m, n)$ and $\Phi(x, y)$ is exactly represented by (1).

\section{Incomplete sampling}

Only the function and its first derivatives will be available in most cases. The omission of $\Phi_{x y}(k \Delta x, l \Delta y)$ from (1) leads to the transform

$$
\begin{aligned}
M N \hat{F}(m, n) & =M N F(m, n)-\operatorname{sgn}(m) \operatorname{sgn}(n)\{m n F(m n) \\
& +m[n-\operatorname{sgn}(n) N] F[m, n-\operatorname{sgn}(n) N] \\
& +n[m-\operatorname{sgn}(m) M] F[m-\operatorname{sgn}(m) M, n] \\
& +[n-\operatorname{sgn}(n) N][m-\operatorname{sgn}(m) M] F[m-\operatorname{sgn} \\
& \times(m) M, n-\operatorname{sgn}(n) N]\},
\end{aligned}
$$

where the inverse transform

$$
\hat{\Phi}(x, y)=\text { F.T. }^{-1}\{\hat{F}(m, n)\} \neq \Phi(x, y)
$$

for arbitrary $(x, y)$ unless $\Phi_{x y}(x, y)=0$. However, there is a special case of interest. For the particular choices $x=k \Delta x, y=\left(l \pm \frac{1}{2}\right) \Delta y$ and $x=\left(k \pm \frac{1}{2}\right) \Delta x, y=l \Delta y$, $\hat{\Phi}$ reduces to $\Phi$. That is, $\Phi$ is given exactly at intervals of $\Delta x / 2$ and $\Delta y / 2$ along lines connecting the original grid points. As a consequence, waves of length $\Delta x$ (or $\Delta y$ ) can be defined along these mesh lines. The points $x=\left(k \pm \frac{1}{2}\right) \Delta x, y=\left(l \pm \frac{1}{2}\right) \Delta y$ are not given correctly unless $\Phi_{x y}(x, y)$ vanishes, or is stipulated.

\section{Conclusions}

Under the geostrophic assumption, simultaneous observations of geopotential and horizontal wind on the grid $[k \Delta x, l \Delta y]$ define exact field values on the refined net consisting of the points $\left[\left(k \pm \frac{1}{2}\right) \Delta x, l \Delta y\right]$ and $\left[k \Delta x,\left(l \pm \frac{1}{2}\right) \Delta y\right]$. For a given $y=l \Delta y, x$-direction waves of length $\Delta x$ may be resolved. A similar result obtains for fixed $x=k \Delta x$. Thus, if the variance spectrum of $\Phi(x, y)$ has no significant contributions from $x$-direction waves shorter than $\Delta x$, it can be reconstituted along lines given by $y=l \Delta y$.

Aside from variations in the Coriolis parameter, if independent horizontal shear estimates can be made, band-limited fields can be determined exactly through the geostrophic relation linking wind and geopotential.

Although no band-limited fields would be expected in practice, the incorporation of derivative information does reduce the aliasing ambiguity by virtue of decreasing the effective minimum definable wavelengths. Further, given an $a$ priori estimate of the variance spectra, the sampling interval and choice of fields to be observed should be considered in light of the appropriate sampling theorem in any experimental design. The results shown here are intended for the case of geostrophic flow, but they are applicable to any holonomic constraint involving first derivatives.

Errors have not been considered. If present, they are incorporated with the true field. Also, since observations ordinarily are not made on a regular net, the applicability of these results for the case of irregularly distributed observation sites must be demonstrated.

\section{REFERENCES}

Linden, D. A., and N. M. Abramson, 1960: A generalization of the Sampling Theorem. Information Control, 3, 26-31.

Sasaki, Y., 1958: An objective analysis based on the variational method. J. Meteor. Soc. Japan, 36, 77-88.

Stephens, J. J., 1966: A variational approach to numerical weather analysis and prediction. Ph.D. dissertation, Texas A\&M University, College Station. 